\title{
Prevalence and Gender Differences of Metabolic Syndrome in Young Ketosis-Prone Type 2 Diabetic Individuals: A Retrospective Study
}

This article was published in the following Dove Press journal:

Diabetes, Metabolic Syndrome and Obesity: Targets and Therapy

\author{
Shichun $\mathrm{Du}^{\mathrm{l}, *}$ \\ Hongmei Zhang ${ }^{1, *}$ \\ Hui $\mathrm{Wu}^{2}$ \\ Shu $\mathrm{Ye}^{1}$ \\ Wen $\mathrm{Li}^{1}$ \\ Qing Su'
}

'Department of Endocrinology, Xin Hua Hospital Affiliated to Shanghai Jiao Tong University School of Medicine, Shanghai 200092, People's Republic of China;

${ }^{2}$ Department of Endocrinology, Zhejiang Provincial People's Hospital, Hangzhou

Medical College, Hangzhou 3100I4,

People's Republic of China

*These authors contributed equally to this work
Correspondence: Qing Su

Department of Endocrinology, Xin Hua Hospital Affiliated to Shanghai Jiao Tong University School of Medicine, Kong Jiang Road 1665\#, Shanghai 200092, People's

Republic of China

$\mathrm{Tel} / \mathrm{Fax}+862125077538$

Email suqing@xinhuamed.com.cn
Purpose: This study aimed to identify the prevalence and gender differences of metabolic syndrome in young new-onset ketosis-prone type 2 diabetic (KPT2D) individuals.

Patients and Methods: A retrospective study was conducted in Shanghai Xin Hua Hospital from 2007 to 2019. A total of 304 patients from 12 to 40 years of age with newly diagnosed diabetes presenting with ketosis were analyzed. The clinical features and laboratory results of KPT2D and type 1 diabetic (T1D) individuals were compared. Prevalence and gender differences of metabolic syndrome in the KPT2D subjects were analyzed.

Results: The prevalence of metabolic syndrome $(P<0.0001)$ was significantly higher in young KPT2D than T1D subjects. The prevalence of high blood pressure $(P<0.0001)$, central obesity $(P<0.0001)$, low plasma HDL-C concentration $(P=0.045)$, and hypertriglyceridemia $(P<0.0001)$ was elevated in the KPT2D subjects compared with T1D. Male predominance $(89 \%)$ was presented in the KPT2D subjects. The prevalence of metabolic syndrome $(P=0.0002)$ was significantly higher in young male than female KPT2D subjects. The presence of central obesity $(P<0.0001)$ and high blood pressure $(P=0.03)$ was higher in male KPT2D subjects than female. The presence of serum triglyceride concentrations $\geq$ $2.3 \mathrm{mmol} / \mathrm{L}$ was significantly higher $(P=0.011)$ in male KPT2D subjects than female.

Conclusion: Significantly higher prevalence of metabolic syndrome in young KPT2D patients compared with T1D patients could be an important reference for diabetic differential diagnosis. KPT2D presented a higher predominance in young males, who had higher prevalence of metabolic syndrome than young females.

Keywords: ketosis, glucose, overweight, hyperglycemia

\section{Introduction}

Traditionally, ketosis and ketoacidosis have been considered acute complications of type 1 diabetes (T1D) in young patients. ${ }^{1,2}$ However, due to the changes in diet and lifestyle, recent evidence has indicated that the number of cases of ketosis-prone type 2 diabetes (KPT2D) will likely increase rapidly in China. ${ }^{3-7}$ Furthermore, KPT2D has also been increasingly reported in the pediatric population. ${ }^{3-7}$ KPT2D is characterized by diabetes that presents with unprovoked ketosis or ketoacidosis but does not necessarily have the typical phenotype of T1D. ${ }^{5,6,8,9}$ Because of the young-onset and ketosis-prone features, confusions exist among clinicians in regard to the differential diagnosis and classifications of these symptoms and subsequent treatments. Some researchers have investigated a high incidence of metabolic 
syndrome among KPT2D individuals than T1D in adults. ${ }^{10,11}$ However, data are limited concerning the relationship between metabolic syndrome and KPT2D in young. To improve glucose control and optimize risk factor managements, this study aimed to identify the characteristics of new-onset KPT2D in young individuals by comparing with those of TID individuals and by describing the gender differences of metabolic syndrome in young KPT2D individuals.

\section{Patients and Methods Subjects}

Our data were obtained from diabetic patients admitted to the Endocrinology Department of Xin Hua Hospital affiliated with Shanghai Jiao Tong University School of Medicine from May 2007 to July 2019. A retrospective medical record review of all episodes of new-onset diabetes from 12 to 40 years of age was performed. Diabetes was diagnosed according to the 2006 America Diabetes Association diagnostic criteria. ${ }^{12}$ Ketosis-prone type 2 diabetes was defined by preserved $\beta$-cell function as fasting C-peptide $>0.33 \mathrm{nmol} / \mathrm{L}$ and negative islet cell antibodies (ICAs), glutamate decarboxylase autoantibodies (GADs), and insulin autoantibodies (IAAs). Furthermore, the new-onset type 2 diabetic patients were admitted to the hospital with ketosis (urine ketone body $\geq 50 \mathrm{mg} / \mathrm{dL}$ or capillary $\beta$-hydroxybutyrate $>1 \mathrm{mmol} / \mathrm{L}$ with hyperglycemia) or diabetic ketoacidosis (DKA, with arterial blood $\mathrm{pH}$ $<7.30$ or serum bicarbonate $<18 \mathrm{mmol} / \mathrm{L}$ apart from ketosis and hyperglycemia ${ }^{13}$ ) as the first symptom, but without obvious precipitating causes, such as infection, stress, surgery, and trauma. T1D was diagnosed by fasting C-peptide $<0.33 \mathrm{nmol} / \mathrm{L}$ and positive autoimmune GADs, IAAs, or ICAs on initial admission. We excluded the patients of prolonged fasting or alcohol ingestion by investigating patients' personal history. Patients who were pregnant, had pancreatic exocrine diseases, or endocrine diseases caused secondary to diabetes were excluded. Patients with malignant tumor or serious dysfunction of liver, renal, heart, lung were excluded. This study was approved by the Human Research Ethics Committee of Xin Hua Hospital affiliated with Shanghai Jiao Tong University School of Medicine and a waiver of consent obtained because of the retrospective nature of the study. All the patients' data are covered confidentiality in Xin Hua Hospital and in compliance with the Declaration of Helsinki.

\section{Definition of Metabolic Syndrome (MetS) Subjects}

According to the revised National Cholesterol Education Program Adult Treatment Panel (NCEP ATP) III criteria for Asia, ${ }^{14}$ the diagnosis of MetS was based on the existence of three or more of the following components: 1 . central obesity, with waist circumference $\geq 90 \mathrm{~cm}$ for males or $80 \mathrm{~cm}$ for females; 2. plasma triglyceride level $\geq 1.7 \mathrm{mmol} / \mathrm{L} ; 3$. high-density lipoprotein cholesterol level $<1.03 \mathrm{mmol} / \mathrm{L}$ in males or $<1.3 \mathrm{mmol} / \mathrm{L}$ in females, or treatment for dyslipidemia; 4. systolic blood pressure $\geq 130 \mathrm{mmHg}$, diastolic blood pressure $\geq 85 \mathrm{mmHg}$, or treatment for hypertension; 5 . high fasting plasma glucose $\geq 5.6 \mathrm{mmol} / \mathrm{L}$.

\section{Clinical Data Collection}

All patients received physical and biochemical examinations after admission to the hospital. Weight and height were measured in light clothing. Waist circumference was measured at the midpoint between the lower margin of the last palpable rib and the top of the iliac crest. ${ }^{15}$ Hip circumference was measured around the widest portion of the buttocks. ${ }^{15}$ The waist to hip ratio (WHR) was calculated as waist measurement divided by hip measurement. Body mass index (BMI) was calculated by dividing weight $(\mathrm{kg})$ by height squared $\left(\mathrm{m}^{2}\right)$. Blood pressure was measured twice using a standard mercury sphygmomanometer after the subject had been seated for at least $5 \mathrm{~min}$. All fasting venous blood samples were taken in the morning following overnight fasting for at least $8 \mathrm{~h}$. Serum total cholesterol, triglyceride, low-density lipoprotein cholesterol (LDLcholesterol) and high-density lipoprotein cholesterol (HDLcholesterol), alanine aminotransferase (ALT), aspartate aminotransferase (AST), creatinine, and uric acid were measured using a fully automatic biochemical analyzer (Hitachi 7600 , Japan). Glycosylated hemoglobin A1c (HbA1c) was measured by high-performance liquid chromatography with an automated biochemistry analyzer (Bio-Rad Variant II, USA). Urine acetone bodies were measured using chemical analysis. Capillary $\beta$-hydroxybutyrate was measured using Abbott test strips. Plasma C-peptide was determined with a chemiluminescence analyzer (Roche e601, USA).

\section{Statistical Analysis}

Data were analyzed with JMP 9.0 (SAS Institute). Twotailed $P$ values $<0.05$ were considered statistically significant. Continuous variables were presented as the mean \pm 
Table I Anthropometric and Clinical Features of the Study Subjects

\begin{tabular}{|c|c|c|c|c|}
\hline & KPT2D & TID & $\mathbf{P}$ & P Adjusted \\
\hline Subjects & 189 & 115 & - & - \\
\hline Age $(y r)$ & $29 \pm 0.7$ & $23 \pm 0.7$ & $<0.0001$ & 0.003 \\
\hline Gender (male n, \%) & $168,89 \%$ & $52,45 \%$ & $<0.0001$ & - \\
\hline Diabetic symptom duration (months) & $2.8 \pm 0.3$ & $1.1 \pm 0.2$ & $<0.0001$ & $<0.0001$ \\
\hline Diabetic family history ( $\mathrm{n}, \%)$ & $115,61 \%$ & $25,22 \%$ & $<0.0001$ & $<0.0001$ \\
\hline Smoking or alcohol drinking habits ( $\mathrm{n}, \%)$ & $98,52 \%$ & $6,5 \%$ & $<0.0001$ & $<0.0001$ \\
\hline Height $(\mathrm{cm})$ & $176 \pm 1.6$ & $166 \pm 2.1$ & $<0.0001$ & 0.005 \\
\hline Weight (kg) & $89 \pm 2.5$ & $53 \pm 4.2$ & $<0.0001$ & $<0.0001$ \\
\hline Body mass index $\left(\mathrm{kg} / \mathrm{m}^{2}\right)$ & $29 \pm 0.3$ & $19 \pm 0.4$ & $<0.0001$ & $<0.0001$ \\
\hline Waist circumference $(\mathrm{cm})$ & $101 \pm 1.3$ & $73 \pm 1.9$ & $<0.0001$ & $<0.0001$ \\
\hline Hip circumference $(\mathrm{cm})$ & $104 \pm 1.2$ & $78 \pm 3.0$ & $<0.0001$ & $<0.0001$ \\
\hline Waist to hip ratio & $0.97 \pm 0.01$ & $0.93 \pm 0.02$ & $<0.0001$ & 0.01 \\
\hline Systolic pressure $(\mathrm{mmHg})$ & $133 \pm 1.9$ & $116 \pm 2.5$ & $<0.0001$ & $<0.0001$ \\
\hline Diastolic pressure $(\mathrm{mmHg})$ & $85 \pm 1.4$ & $73 \pm 2.0$ & $<0.0001$ & $<0.0001$ \\
\hline
\end{tabular}

Notes: Data are presented with means \pm SEM or \%; $P$, KPT2D versus TID; $P$ adjusted, KPT2D versus TID adjusted by gender. Abbreviations: KPT2D, ketosis-prone type 2 diabetes; TID, type I diabetes with ketosis.

SEM and were analyzed with $t$-test or ANOVA, and nonnormally distributed variables (triglycerides, ALT, and C-peptide) were log-transformed to be analyzed. A chisquare test or Fisher exact test was used to determine the frequencies in categorical variables. Univariate and multivariate logistic regression were performed to evaluate risk factors associated with MetS in KPT2D subjects. The multivariate analysis controlled for all variables with significance from univariate analyses.

\section{Results}

\section{Anthropometric and Clinical Characteristics of the Study Subjects}

Totally, 482 cases of newly-onset diabetic patients with ketosis from 12 to 40 years were selected. Among them, 55 cases were excluded with obvious precipitating causes, such as infection, stress, surgery, and trauma, and 27 cases were excluded with prolonged fasting or alcohol ingestion by investigating patients' personal history. Then, 31 cases with disturbance of consciousness, 22 patients who were pregnant, 8 with pancreatic exocrine diseases, 9 with endocrine diseases caused secondary to diabetes, 20 with serious dysfunction of lung, liver, heart, or renal, and 6 with malignant tumor were excluded. At last, 304 cases were analyzed. The anthropometric and clinical features of the study population are presented in Table 1. A total of 304 cases of newly diagnosed diabetic patients presenting with ketosis from 12 to 40 years of age were analyzed. The KPT2D population consisted of $168(89 \%)$ men and 21
(11\%) women with a mean age of $29 \pm 0.7$ years. The T1D population consisted of $52(45 \%)$ men and $63(55 \%)$ women with a mean age of $23 \pm 0.7$ years. Since male predominance was observed in the KPT2D population, which was in accordance with previous observations, ${ }^{16-18}$ all subsequent statistical analyses were performed with a gender correction. Unlike T1D, patients with KPT2D had a strong diabetic family history (61\%), smoking, or alcohol consumption ( $52 \%$, mainly in the older half of the age ranges). Statistically significant differences in other comparisons between the two groups were found, including BMI, waist and hip circumference, and blood pressure, as expected.

\section{Laboratory Results of the Study Subjects}

Laboratory data findings of the groups are presented in Table 2. There were no significant differences in plasma fasting glucose concentration $(13.3 \pm 0.3$ vs $13.0 \pm 0.8 \mathrm{mmol} / \mathrm{L}$, respectively, in the KPT2D and T1D groups, $P=0.90$ adjusted for gender), $2 \mathrm{~h}$ postprandial glucose concentration (19.7 \pm 0.5 vs $18.4 \pm 0.9 \mathrm{mmol} / \mathrm{L}$, respectively, in the KPT2D and T1D groups, $P=0.08$ adjusted for gender) or $\mathrm{HbA1c}$ (11.8 \pm 0.3 vs $12.7 \pm 0.4 \%$, respectively, in the KPT2D and T1D groups, $P=0.08$ adjusted for gender) levels between the two groups with diabetes on admission. However, the fasting C-peptide $(0.66 \pm 0.03$ vs $0.12 \pm 0.02 \mathrm{nmol} / \mathrm{L}$, respectively, in the KPT2D and T1D groups, $P<0.0001$ adjusted for gender) and $2 \mathrm{~h}$ postprandial $\mathrm{C}$-peptide concentration (1.02 \pm 0.06 vs $0.19 \pm 0.06 \mathrm{mmol} / \mathrm{L}$, respectively, in the KPT2D and T1D groups, $P<0.0001$ adjusted by gender), which both measure 
Table 2 Laboratory Data Findings of the Study Subjects

\begin{tabular}{|c|c|c|c|c|}
\hline & KPT2D & TID & $\mathbf{P}$ & P Adjusted \\
\hline \multicolumn{5}{|l|}{ Glucose } \\
\hline Fasting glucose $(\mathrm{mmol} / \mathrm{L})$ & $13.3 \pm 0.3$ & $13.0 \pm 0.8$ & 0.93 & 0.90 \\
\hline $2 \mathrm{~h}$ glucose $(\mathrm{mmol} / \mathrm{L})$ & $19.7 \pm 0.5$ & $18.4 \pm 0.9$ & 0.19 & 0.08 \\
\hline Fasting C-peptide (nmol/L) & $0.66 \pm 0.03$ & $0.12 \pm 0.02$ & $<0.0001$ & $<0.0001$ \\
\hline 2 h C-peptide (nmol/L) & $1.02 \pm 0.06$ & $0.19 \pm 0.06$ & $<0.0001$ & $<0.0001$ \\
\hline Hemoglobin Alc (\%) & $11.8 \pm 0.3$ & $12.7 \pm 0.4$ & 0.03 & 0.08 \\
\hline \multicolumn{5}{|l|}{ Acidosis } \\
\hline Ketoacidosis proportion (n, \%) & $57,30 \%$ & $62,54 \%$ & $<0.0001$ & $<0.0001$ \\
\hline $\mathrm{PH}$ & $7.20 \pm 0.02$ & $7.12 \pm 0.02$ & 0.005 & 0.011 \\
\hline $\mathrm{HCO} 3(\mathrm{mmol} / \mathrm{L})$ & $11.7 \pm 0.5$ & $8.2 \pm 0.5$ & 0.039 & 0.049 \\
\hline \multicolumn{5}{|l|}{ Lipids } \\
\hline Total cholesterol $(\mathrm{mmol} / \mathrm{L})$ & $4.8 \pm 0.2$ & $4.0 \pm 0.2$ & 0.01 & 0.005 \\
\hline Triglyceride $(\mathrm{mmol} / \mathrm{L})$ & $3.9 \pm 0.3$ & $1.0 \pm 0.3$ & $<0.0001$ & 0.002 \\
\hline HDL-cholesterol (mmol/L) & $1.07 \pm 0.04$ & $1.39 \pm 0.05$ & 0.005 & 0.02 \\
\hline LDL-cholesterol $(\mathrm{mmol} / \mathrm{L})$ & $2.69 \pm 0.10$ & $2.59 \pm 0.17$ & 0.67 & 0.13 \\
\hline \multicolumn{5}{|l|}{ Liver enzymes } \\
\hline Alanine aminotransferase $(\mathrm{U} / \mathrm{L})$ & $63.5 \pm 5.0$ & $18.75 \pm 6.3$ & 0.0001 & 0.0004 \\
\hline Aspartate aminotransferase (U/L) & $46.8 \pm 5.7$ & $23.9 \pm 6.8$ & 0.013 & 0.04 \\
\hline \multicolumn{5}{|l|}{ Renal function } \\
\hline Serum creatinine $(\mu \mathrm{mol} / \mathrm{L})$ & $83.7 \pm 3.5$ & $86.9 \pm 3.6$ & 0.38 & 0.81 \\
\hline Serum uric acid $(\mu \mathrm{mol} / \mathrm{L})$ & $377 \pm 14$ & $210 \pm 16$ & $<0.0001$ & 0.001 \\
\hline eGFR $\left(\mathrm{mL} / \mathrm{min} / \mathrm{l} .73 \mathrm{~m}^{2}\right)$ & $105 \pm 0.5$ & $103 \pm 0.5$ & 0.76 & 0.77 \\
\hline
\end{tabular}

Notes: Data are presented with means \pm SEM or \%; $P$, KPT2D versus TID; $P$ adjusted, KPT2D versus TID adjusted by gender. Abbreviations: KPT2D, ketosis-prone type 2 diabetes; TID, type I diabetes with ketosis.

pancreatic $\beta$-cell function, were found to be higher in KPT2D patients than in T1D patients. The plasma triglyceride (TG) level was significantly elevated in KPT2D compared with T1D $(3.9 \pm 0.3$ vs $1.0 \pm 0.3 \mathrm{mmol} / \mathrm{L}$, respectively, in the KPT2D and T1D groups, $P=0.002$ adjusted for gender). Total cholesterol levels were elevated $(P=0.005$ adjusted for gender) and HDL-cholesterol levels were decreased $(P=$ 0.02 adjusted for gender) in the KPT2D group, while LDLcholesterol and were not different between the two groups. Serum ALT $(P=0.0004), \operatorname{AST}(P=0.04)$, and uric acid $(P=$ $0.001)$ were found to be significantly elevated in the KPT2D group, when adjusted for gender.

\section{The Prevalence of MetS in Ketosis-Prone Type 2 Diabetic Subjects}

The presence and characteristics of MetS constituents in KPT2D patients are shown in Figure 1. The prevalence of metabolic syndrome in KPT2D and T1D subjects was $81.5 \%$ and $18.3 \%$, respectively, $P<0.0001$, OR 19.7, 95\% CI 10.8-35.8. The distribution of MetS components in KPT2D subjects included three MetS components
$(11.6 \%, \mathrm{n}=22)$, four MetS components $(35.4 \%, \mathrm{n}=67)$ and five MetS components $(34.4 \%, \mathrm{n}=65)$. Furthermore, we analyzed each component. The prevalence of high blood pressure (41.3 vs $10.4 \%$, respectively, in the KPT2D and T1D groups, $P<0.0001)$, central obesity ( 83.0 vs $17.4 \%$, respectively, in the KPT2D and T1D groups, $P<0.0001$ ), low plasma HDL-C concentration (42.9 vs 31.3\%, respectively, in the KPT2D and T1D groups, $P=0.045$ ), and hypertriglyceridemia (67.7 vs $12.2 \%$, respectively, in the KPT2D and T1D groups, $P<$ $0.0001)$ were elevated in KPT2D subjects.

\section{The Gender Differences of MetS in}

\section{Ketosis-Prone Type 2 Diabetic Subjects}

Figure 2 shows the gender differences of MetS in ketosisprone type 2 diabetic subjects. The prevalence of metabolic syndrome in male and female subjects was $85.7 \%$ and $47.6 \%$, respectively, $P=0.0002$, OR $6.6,95 \%$ CI 2.5-17.2. Furthermore, we compared the gender difference of each component of MetS in KPT2D subjects. The prevalence of high blood pressure (44.1 vs $19.0 \%$, respectively, 

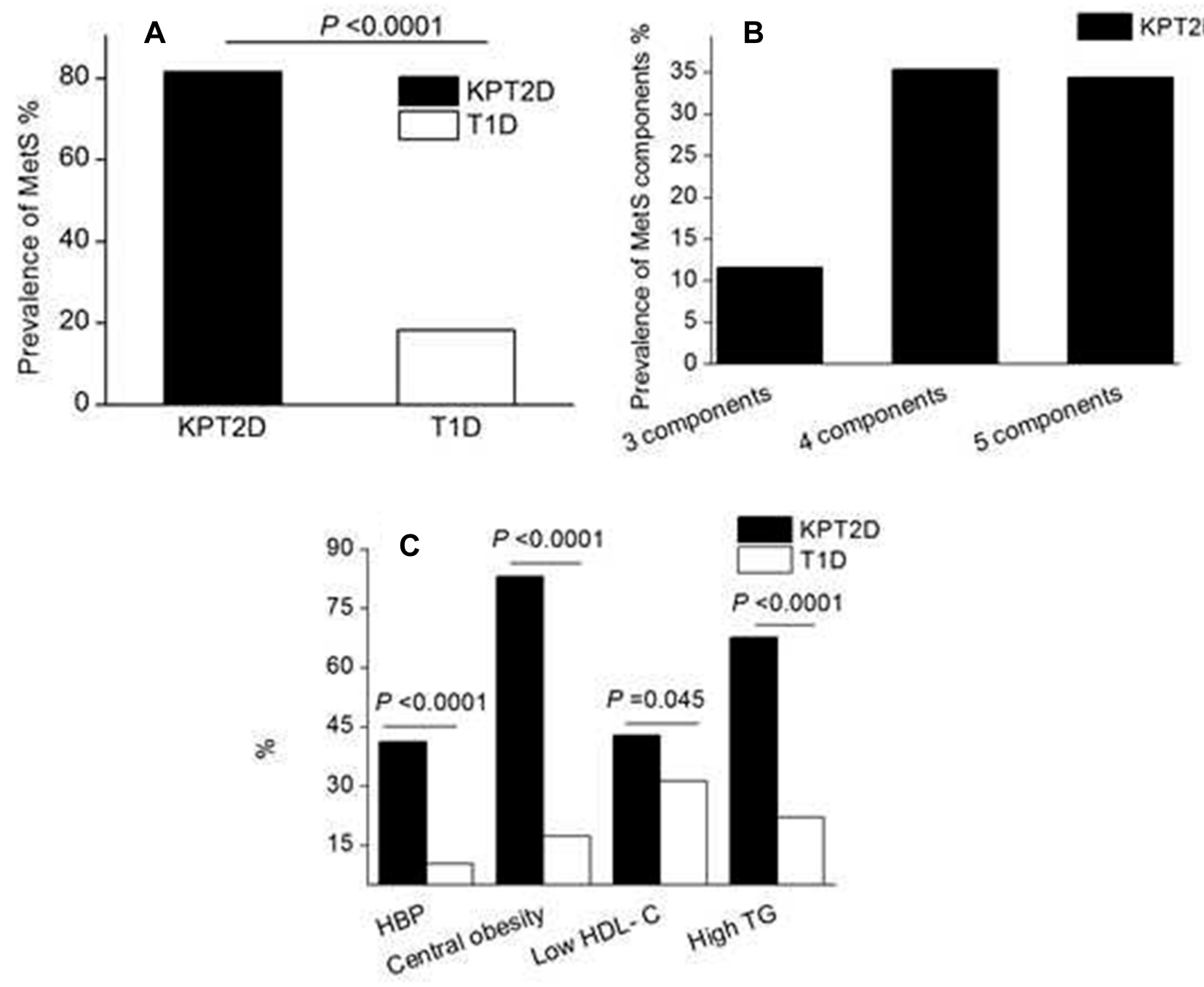

Figure I Prevalence of metabolic syndrome (MetS) and components in ketosis-prone type 2 diabetic (KPT2D) and type I diabetic (TID) subjects. Notes: The prevalence of MetS (A), distribution of MetS components (B), and each component (C) in KPT2D and TID subjects.

in the male and female KPT2D subjects, $P=0.03$; OR 3.3, $95 \%$ CI $1.1-10.4)$ and central obesity ( 88.1 vs $42.9 \%$, respectively, in the male and female KPT2D subjects, $P<$ 0.0001; OR 9.9, 95\% CI 3.7-26.4) were higher in male than female KPT2D subjects. Hypertriglyceridemia (69.6 vs $52.4 \%$, respectively, in the male and female KPT2D subjects, $P=0.11$; OR $2.1,95 \%$ CI $0.8-5.2)$ and low plasma HDL-C concentration (43.5 vs $33.3 \%$, respectively, in the male and female KPT2D subjects, $P=0.38$; OR $1.5,95 \%$ CI 0.6-4.0) were not different between sex. However, the prevalence of serum TG concentration $\geq 2.3 \mathrm{mmol} / \mathrm{L}$ was significantly elevated in male than female ( 48.2 vs $19.0 \%$, $P=0.011$; OR $1.0,95 \%$ CI 1.3-12.3) with TG concentration $\geq 5.6 \mathrm{mmol} / \mathrm{L}$ among $21.4 \%$ of the male KPT2D patients and TG concentration $\geq 11.2 \mathrm{mmol} / \mathrm{L}$ among $8.3 \%$ of the male KPT2D patients.
Table 3 provides the risk factors for MetS by logistic regression analyses in KPT2D subjects. Gender $(P=$ 0.0002), BMI $(P<0.0001)$, serum ALT $(P=0.01)$, HbA1C $(P=0.028)$, and serum UA $(P=0.045)$ were associated with MetS in KPT2D subjects in univariate logistic regression analyses. After adjusting all above variables by multivariate logistic regression analyses, however, only gender difference $(P=0.006)$ and BMI $(P<0.0001)$ continued to exist as risk factors for MetS in KPT2D subjects.

\section{Discussion}

Ketosis-prone type 2 diabetes was first described in young African Americans and sub-Saharan Africans. ${ }^{19,20}$ The patients often presented with remarkable hyperglycemia, ketosis, or even ketoacidosis, as well as reversible $\beta$-cell dysfunction. ${ }^{20,21}$ In the past two or three decades, clinical 

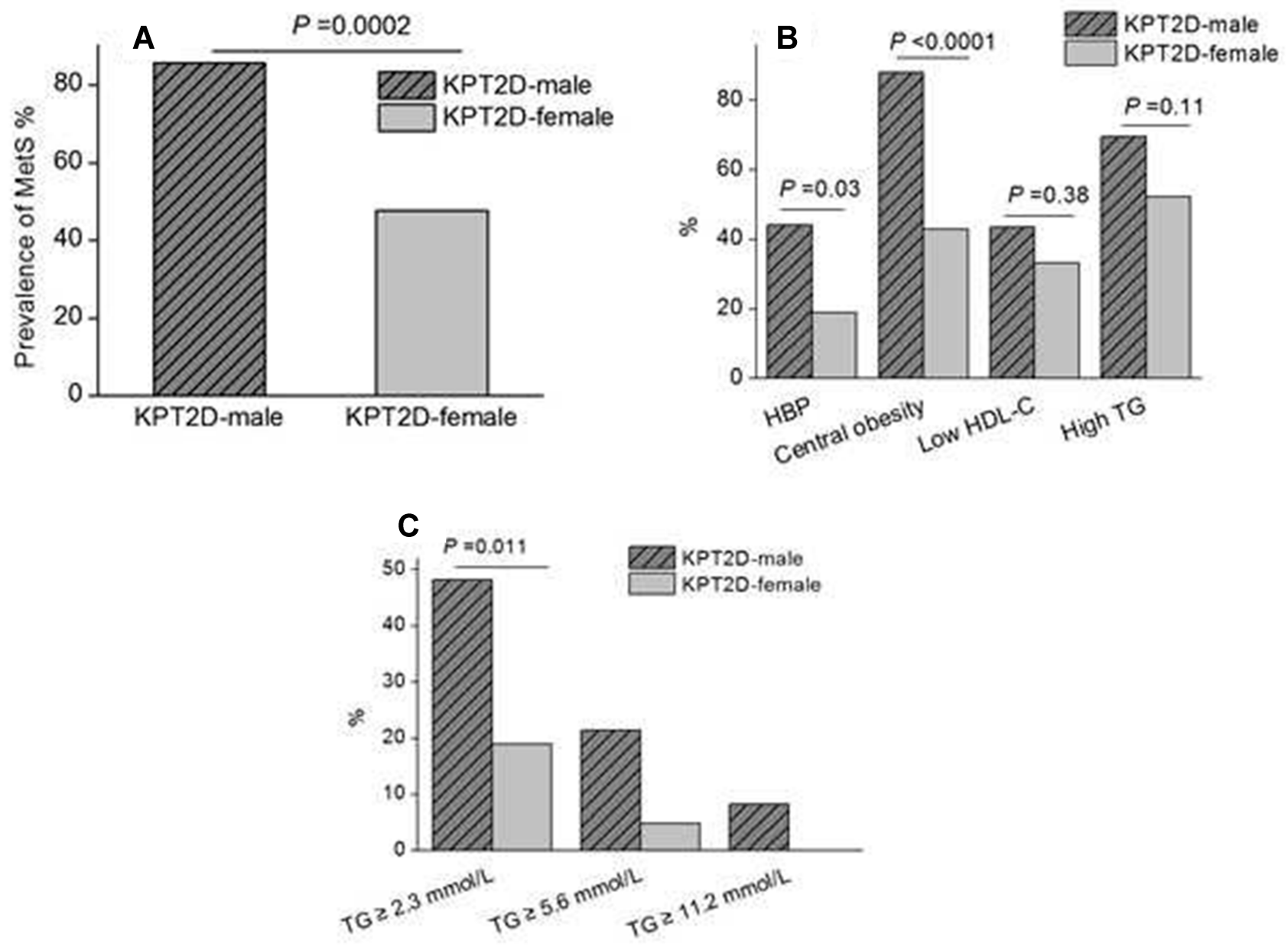

Figure 2 Gender differences of metabolic syndrome (MetS) and components in ketosis-prone type 2 diabetic (KPT2D) subjects.

Notes: The prevalence of MetS (A), each MetS component (B), and different levels of serum triglyceride (TG) (C) in both male and female KPT2D subjects.

presentations of the disease have been increasingly recognized worldwide. Overweight and obese males seemed to be the most vulnerable to this form of diabetes. ${ }^{18,22}$

In our study, $89 \%$ of the individuals were male, $78 \%$ were overweight or obese. The data were consistent with most $^{20,23}$ but not all prior studies. We found young subjects with KPT2D usually had a strong family history of diabetes. All of these features of KPT2D patients have provided evidence that KPT2D might be a subtype of type 2 diabetes. ${ }^{23}$ However, data were limited about the association of metabolic syndrome and KPT2D in young individuals. Since the classifications of newly diagnosed young-onset ketosis diabetic patients were often confused by clinicians, the presence of MetS might help to

Table 3 Risk Factors for Metabolic Syndrome by Logistic Regression Analyses in Ketosis-Prone Type 2 Diabetic Subjects

\begin{tabular}{|l|l|l|l|l|}
\hline \multirow{2}{*}{ Variables } & \multicolumn{2}{|l|}{ Univariate } & \multicolumn{2}{l|}{ Multivariate } \\
\cline { 2 - 5 } & OR (95\% Cl) & P & OR (95\% Cl) & $P$ \\
\hline Gender (male) & $6.7(2.5-18.5)$ & 0.0002 & $8.3(2.0-25.1)$ & 0.006 \\
Body mass index & $2.8(1.9-5.5)$ & $<0.0001$ & $3.2(1.9-7.6)$ & $<0.0001$ \\
ALT & $1.02(1.01-1.04)$ & 0.01 & $1.03(1.0-1.1)$ & 0.05 \\
Hemoglobin Alc & $1.4(1.1-1.7)$ & 0.0028 & $1.2(0.7-2.4)$ & 0.49 \\
Serum uric acid & $1.01(1.0-1.01)$ & 0.045 & $1.00(1.0-1.0)$ & 0.92 \\
\hline
\end{tabular}

Abbreviations: univariate, univariate logistic regression analyses; multivariate, multivariate logistic regression analyses; OR, odds ratio; $\mathrm{Cl}$, confidence interval; $\mathrm{ALT}$, serum alanine aminotransferase. 
distinguish patients with ketosis-prone diabetes who had a type 2 diabetic phenotype. ${ }^{10}$ In our study, we found that the prevalence of MetS and its components were significantly elevated in young ketosis-prone type 2 diabetic subjects. Young KPT2D individuals showed a 2- to 3 -fold higher prevalence of metabolic syndrome compared with that of a previous population-based investigation. ${ }^{24}$ And most subjects suffered from 4 to 5 components of MetS, which demonstrated the severity of metabolic chaos in KPT2D individuals. Male KPT2D individuals had a significantly higher prevalence of high blood pressure, central obesity, and hypertriglyceridemia (TG concentration $\geq 2.3 \mathrm{mmol} / \mathrm{L}$ ) compared with female in the KPT2D subject. Furthermore, we found serum TG concentration $\geq$ $5.6 \mathrm{mmol} / \mathrm{L}$ among $21.4 \%$ and $\mathrm{TG}$ concentration $\geq$ $11.2 \mathrm{mmol} / \mathrm{L}$ among $8.3 \%$ of the male KPT2D patients, which were not detected by previous investigators. The percentages were even 10- and 20-fold higher than those of a previous population-based investigation. ${ }^{25}$ Elevated levels of plasma triglyceride were usually associated with atherosclerosis and cardiovascular disease, even in the absence of high total plasma cholesterol levels. ${ }^{26}$ Severely elevated plasma triglyceride levels even increased the risk of acute pancreatitis. Further long-term follow-up and prospective studies are needed to investigate the presence of cardiovascular disease and pancreatitis in these populations during our future investigations.

For many years, gender differences in metabolic syndrome were attributed to gonadal hormones. ${ }^{27,28}$ It has been well recognized that men accumulated adipose tissue in the visceral area to a much greater extent than women. Gender differences in body fat distribution as well as its associated insulin sensitivity have been suggested to partly explain the different metabolic profiles and ketosis risk in young type 2 diabetic patients. ${ }^{29}$ Apart from gonadal hormones, the gut microbiome and environmental factors such as diet, endocrine pollutants, and circadian cycle might affect metabolism differently between males and females. ${ }^{27,28}$

Wang et al have investigated the prevalence of metabolic syndrome in ketosis-onset diabetes (KOD). ${ }^{11}$ However, the concept of KPT2D is not the same with KOD. Liu et $\mathrm{al}^{5}$ indicated that KOD and KPD do not absolutely have the same clinical characteristics. The enrolled criteria of KPT2D subjects in our study meet both the diagnosis of T2D and ketosis-onset rather than only ketosis-onset. Besides, our study focused on adolescence and young adults, while Wang et al investigated adults older than 17 years. We focused on young individuals not only because confusions always existed about classifications of new-onset diabetes in young patients by clinicians but also the high prevalence of MetS might promote the development of endothelial dysfunction and atherosclerosis in the long term. ${ }^{26}$ Because of rapid increasing incidence of MetS in adolescent and young adults, we should pay more attention to established risk factors and suggest lifestyle interventions at an earlier period. ${ }^{30}$ Further studies would be helpful for investigating the impact of MetS to atherosclerotic risks in these young KPT2D subject.

Some major limitations of the present study should be addressed. First, no general consensus existed regarding the definitions of MetS in China. We have adopted the revised NCEP ATP III Criteria for Asians ${ }^{14}$ with a revised waist circumference cut-off of $\geq 90 \mathrm{~cm}$ (males) and $\geq 80 \mathrm{~cm}$ (females) since Asians are known to develop cardiometabolic risk at lower thresholds for waist circumference. This revised NCEP ATP III Criteria has been adopted by many Chinese studies. ${ }^{31-}$

${ }^{33} \mathrm{Lu}$ et $\mathrm{al}^{33}$ have developed a study with a sample of 98,658 Chinese adults covering all 31 provinces of China using this Criteria. Second, the single-center and retrospective observational study might have bias. However, we controlled the confounding factors as far as possible. Third, we focused on young KPT2D individuals aged from 12 to 40 years, so the data might not be applicable to a wide range of ages. Fourth, we did not mention general stage of sexual maturity for the adolescents who may still be growing and advancing in puberty. However, none of the MetS definitions consider the influences in puberty including the changes in insulin resistance, in fat and fat-free mass, as well as the secretion of growth and sex steroid hormone. ${ }^{34}$

Therefore, knowledge of the characteristics and traits of MetS in young KPT2D patients is crucial for helping diagnosis and initiating early and individualized treatment. Another important aspect for identifying the gender difference of KPT2D is to focus more attention on investigating the risks of chronic cardiovascular or atherosclerotic disorders in the male subjects in the long term.

\section{Conclusion}

We found that young individuals with ketosis-prone type 2 diabetes had reserved islet $\beta$-cell function compared with T1D individuals. Young individuals with ketosisprone type 2 diabetes had a high incidence of metabolic syndrome. Male presented a high predominance in 
young KPT2D individuals. Meanwhile, male had higher prevalence of metabolic syndrome than female. A better understanding of the metabolic dysfunction of KPT2D will help to identify individuals who have a high risk of cardiovascular complications and to initiate early and aggressive treatment efforts.

\section{Funding}

This work was supported by the National Natural Science Foundation of China (No. 81600702); the research grant of cooperation between medicine and engineering of Shanghai Jiao Tong University (No. YG2015QN43); the research grant of Shanghai Municipal Commission of Health and Family Planning (No. 20144Y0140); the Medical Science and Technology Foundation of Zhejiang Province of China (No. 2014KYA234); and the Foundation of Hangzhou Medical College (No. 2015XZB01).

\section{Disclosure}

The authors report no conflicts of interest in this work.

\section{References}

1. Farsani SF, Brodovicz K, Soleymanlou N, Marquard J, Wissinger E, Maiese BA. Incidence and prevalence of diabetic ketoacidosis (DKA) among adults with type 1 diabetes mellitus (T1D): a systematic literature review. BMJ Open. 2017;7(7):e016587. doi:10.1136/bmjopen2017-016587

2. Tentolouris N, Katsilambros N. Diabetic ketoacidosis in adults. In: Diabetic Emergencies: Diagnosis and Clinical Management. 2011. doi:10.1002/9781119971825.ch1

3. Tan H, Zhou Y, Yu Y. Characteristics of diabetic ketoacidosis in Chinese adults and adolescents - a teaching hospital-based analysis. Diabetes Res Clin Pract. 2012;97(2):306-312. doi:10.1016/j. diabres.2012.05.004

4. Li MF, Ren Y, Zhao CC, et al. Prevalence and clinical characteristics of lower limb atherosclerotic lesions in newly diagnosed patients with ketosis-onset diabetes: a cross-sectional study. Diabetol Metab Syndr. 2014;6(1). doi:10.1186/1758-5996-6-71

5. Liu B, Yu C, Li Q, Li L. Ketosis-onset diabetes and ketosis-prone diabetes: same or not? Int J Endocrinol. 2013;2013:1-6. doi:10.1155/ 2013/821403

6. Lu H, Hu F, Zeng Y, et al. Ketosis onset type 2 diabetes had better islet $\beta$-cell function and more serious insulin resistance. J Diabetes Res. 2014;2014:1-6. doi:10.1155/2014/510643

7. Zhong VW, Juhaeri J, Mayer-Davis EJ. Trends in hospital admission for diabetic ketoacidosis in adults with type 1 and type 2 diabetes in England, 1998-2013: a retrospective cohort study. Diabetes Care. 2018. doi: $10.2337 / \mathrm{dc} 17-1583$

8. Iwasaki Y, Hamamoto Y, Kawasaki Y, et al. Japanese cases of acute onset diabetic ketosis without acidosis in the absence of glutamic acid decarboxylase autoantibody. Endocrine. 2010;37(2):286-288. doi:10. 1007/s12020-009-9301-0

9. Sobngwi E, Gautier JF. Adult-onset idiopathic type I or ketosis-prone type II diabetes: evidence to revisit diabetes classification. Diabetologia. 2002;45(2):283-285. doi:10.1007/s00125-001-0739-8
10. Otiniano ME, Balasubramanyam A, Maldonado M. Presence of the metabolic syndrome distinguishes patients with ketosis-prone diabetes who have a type 2 diabetic phenotype. $J$ Diabetes Complications. 2005;19(6):313-318. doi:10.1016/j.jdiacomp.2005.03.001

11. Wang JW, Wang AP, Chen MY, et al. Prevalence and clinical characteristics of hypertension and metabolic syndrome in newly diagnosed patients with ketosis-onset diabetes: a cross-sectional study. Diabetol Metab Syndr. 2019;11(1):1-9. doi:10.1186/s13098-0190426-x

12. American Diabetes Association. Diagnosis and classification of diabetes mellitus. Diabetes Care. 2006.

13. Kitabchi AE, Umpierrez GE, Miles JM, Fisher JN. Hyperglycemic crises in adult patients with diabetes. Diabetes Care. 2009;32 (7):1335-1343. doi:10.2337/dc09-9032

14. Grundy SM, Brewer HB, Cleeman JI, Smith SC, Lenfant C. Definition of metabolic syndrome: report of the national heart, lung, and blood institute/American heart association conference on scientific issues related to definition. Circulation. 2004. doi:10.1161/01. CIR.0000111245.75752.C6

15. Wang $\mathrm{F}$, Wu S, Song Y, et al. Waist circumference, body mass index and waist to hip ratio for prediction of the metabolic syndrome in Chinese. Nutr Metab Cardiovasc Dis. 2009;19(8):542-547. doi:10.1016/j.numecd.2008.11.006

16. Matsui J, Tamasawa N, Tanabe J, et al. Clinical characteristics of Japanese youth-onset type 2 diabetes with ketonuria. Diabetes Res Clin Pract. 2005;70(3):235-238. doi:10.1016/j.diabres.2005.03.037

17. Das GR, Ramachandran R, Gangadhara P, et al. Clinical characteristics, beta-cell dysfunction and treatment outcomes in patients with A $-\beta+$ ketosis-prone diabetes (KPD): the first identified cohort amongst Asian Indians. J Diabetes Complications. 2017. doi:10.1016/j.jdiacomp.2017.06.008

18. Lontchi-Yimagou E, Nguewa JL, Assah F, et al. Ketosis-prone atypical diabetes in Cameroonian people with hyperglycaemic crisis: frequency, clinical and metabolic phenotypes. Diabet Med. 2017;34 (3):426-431. doi:10.1111/dme.13264

19. Winter WE, Maclaren NK, Riley WJ, Clarke DW, Kappy MS, Spillar RP. Maturity-onset diabetes of youth in black Americans. $N$ Engl J Med. 1987;316(6):285-291. doi:10.1056/NEJM198702 053160601

20. Mauvais-Jarvis F, Sobngwi E, Porcher R, et al. Ketosis-prone type 2 diabetes in patients of sub-saharan african origin: clinical pathophysiology and natural history of $\beta$-cell dysfunction and insulin resistance. Diabetes. 2004;53(3):645-653. doi:10.2337/diabetes.53.3.645

21. Umpierrez GE. Ketosis-prone type 2 diabetes: time to revise the classification of diabetes. Diabetes Care. 2006;29(12):2755-2757. doi: $10.2337 / \mathrm{dc} 06-1870$

22. Yotsapon T, Sarat S. Clinical characteristics and long-term follow-up of ketosis-prone diabetes in thai patients. Exp Clin Endocrinol Diabetes. 2014. doi:10.1055/s-0034-1371812

23. Zhang M, Li Y, Cui W, et al. The clinical and metabolic characteristics of young-onset ketosis-prone type 2 diabetes in China. Endocr Pract. 2015;21(12):1364-1371. doi:10.4158/EP15778.OR

24. Ford ES. Prevalence of the metabolic syndrome defined by the international diabetes federation among adults in the U.S. Diabetes Care. 2005;28(11):2745-2749. doi:10.2337/diacare.28.11.2745

25. Ford ES, Li C, Zhao G, Pearson WS, Mokdad AH. Hypertriglyceridemia and Its pharmacologic treatment among US adults. Arch Intern Med. 2009;169(6):572. doi:10.1001/ archinternmed.2008.599

26. DeFronzo RA, Ferrannini E. Insulin resistance: a multifaceted syndrome responsible for NIDDM, obesity, hypertension, dyslipidemia, and atherosclerotic cardiovascular disease. Diabetes Care. 1991;14 (3):173-194. doi:10.2337/diacare.14.3.173

27. Link JC, Reue K. Genetic basis for sex differences in obesity and lipid metabolism. Annu Rev Nutr. 2017;37(1):225-245. doi:10.1146/ annurev-nutr-071816-064827 
28. Zore T, Palafox M, Reue K. Sex differences in obesity, lipid metabolism, and inflammation - a role for the sex chromosomes? Mol Metab. 2018;15:35-44. doi:10.1016/j.molmet.2018.04.003

29. Wang $X$, Tan H. Male predominance in ketosis-prone diabetes mellitus. Biomed Reports. 2015;3(4):439-442. doi:10.3892/ br.2015.461

30. Kassi E, Pervanidou P, Kaltsas G, Chrousos G. Metabolic syndrome: definitions and controversies. BMC Med. 2011;9(1). doi:10.1186/ 1741-7015-9-48

31. He Y, Li Y, Bai G, et al. Prevalence of metabolic syndrome and individual metabolic abnormalities in China, 2002-2012. Asia Pac J Clin Nutr. 2019. doi:10.6133/apjen.201909_28(3).0023
32. Xi B, He D, Hu Y, Zhou D. Prevalence of metabolic syndrome and its influencing factors among the Chinese adults: the China health and nutrition survey in 2009. Prev Med (Baltim). 2013;57(6):867-871. doi:10.1016/j.ypmed.2013.09.023

33. Lu J, Wang L, Li M, et al. Metabolic syndrome among adults in China: the 2010 China noncommunicable disease surveillance. J Clin Endocrinol Metab. 2017. doi:10.1210/jc.2016-2477

34. Wilson PWF, Umpierrez GE. Insulin resistance and pubertal changes. $J$ Clin Endocrinol Metab. 2008;93(7):2472-2473. doi:10.1210/ jc. $2008-0873$

Diabetes, Metabolic Syndrome and Obesity: Targets and Therapy

\section{Publish your work in this journal}

Diabetes, Metabolic Syndrome and Obesity: Targets and Therapy is an international, peer-reviewed open-access journal committed to the rapid publication of the latest laboratory and clinical findings in the fields of diabetes, metabolic syndrome and obesity research. Original research, review, case reports, hypothesis formation, expert opinion and commentaries are all considered for publication. The manuscript management system is completely online and includes a very quick and fair peer-review system, which is all easy to use. Visit http://www.dovepress.com/testimonials.php to read real quotes from published authors. 